\title{
Internal Transport Barrier Formation and Pellet Injection Simulation in Helical and Tokamak Reactors
}

\author{
You HIGASHIYAMA, Kozo YAMAZAKI, Jeronimo GARCIA ${ }^{1)}$, Hideki ARIMOTO \\ and Tatsuo SHOJI \\ Nagoya University, Furo-cho, Chikusaku, Nagoya 464-8603, Japan \\ ${ }^{1)}$ Universitat Polite 'cnica de Catalunya (UPC), Barcelona, Spain
}

(Received 16 November 2007 / Accepted 17 March 2008)

\begin{abstract}
In the future fusion reactor, plasma density peaking is important for increase in the fusion power gain and for achievement of confinement improvement mode. Density control and internal transport barrier (ITB) formation due to pellet injection have been simulated in tokamak and helical reactors using the toroidal transport linkage code TOTAL. First, pellet injection simulation is carried out, including the neutral gas shielding model and the mass relocation model in the TOTAL code, and the effectiveness of high-field side (HFS) pellet injection is clarified. Second, ITB simulation with pellet injection is carried out with the confinement improvement model based on the $E \times B$ shear effects, and it is found that deep pellet penetration is helpful for ITB formation as well as plasma core fuelling in the reversed-shear tokamak and helical reactors.
\end{abstract}

(C) 2008 The Japan Society of Plasma Science and Nuclear Fusion Research

Keywords: transport simulation, internal transport barrier, pellet injection, tokamak reactor, helical reactor

DOI: $10.1585 /$ pfr.3.S1048

\section{Introduction}

The total fusion reactor power strongly depends on the radial profiles of plasma temperature and density, and density control is important to optimize reactor operation. To control plasma density and pressure profiles, pellet injection is considered a prospective technique. In JET and other tokamak experiments, it was shown that the density profile modifications disagree with pellet ablation theory, which assumes that the pellet particles remain on the magnetic field lines where they are ionized [1-4]. The pellet penetration depth measured using pellet light emission agreed well with pellet ablation theory. This suggested that a fast outward major-radius drift may occur during the pellet ablation and toroidal symmetrization processes. To test this hypothesis, the experiment of high-field side (HFS) pellet injection was performed in ASDEX-Upgrade, and it was shown that the fuelling efficiency and the penetration depth of pellets are improved [5]. Similar results were observed in DIII-D [6] and other tokamak experiments, and HFS pellet injection is expected to be an effective technique of plasma core fuelling in future tokamak reactors.

Transport simulation studies have been carried out focusing on ITB formation in tokamak and helical plasmas. When an ITB is formed in the plasma, it results in good confinement and a significantly peaked pressure profile, so that the operation scenarios with the ITB in tokamaks are expected as enhanced performance modes such as high- $\beta_{\mathrm{p}}$ mode [7], reversed-shear mode [8], and pellet enhanced performance mode (PEP) $[9,10]$. In helical systems the
ITB model based on Bohm and GyroBohm-like transport with $E \times B$ shear flow effects has already been compared with the LHD experimental ITB [11]; this model is inspired from the JET mixed-model [12]. This model is introduced into the toroidal transport linkage TOTAL code $[13,14]$, and is applied to the one-dimensional (1-D) ITB formation simulation of both 3-D equilibrium helical and 2-D equilibrium tokamak plasmas.

Both, pellet injection and ITB formation, have great influence on the density profile and the fusion power output; therefore, we consider an operation scenario with ITB formation by pellet injection density control in helical and tokamak reactor plasmas using the TOTAL code. Section 2 will describe the details of the transport models and the HFS pellet injection model included in the TOTAL code, and simulation results will be shown in section 3 . The conclusion will be given in section 4 .

\section{TOTAL Code}

\subsection{Transport model description}

The Bohm and GyroBohm mixed transport model with the $E \times B$ shear flow effect has already been compared with helical and tokamak experimental ITBs [11, 12]. The most widely accepted explanation for ITB formation relies on the suppression of ITG turbulence due to $E \times B$ shear flow. The suppression of turbulence might occur when the $E \times B$ flow shearing rate $\omega_{E \times B}$ exceeds the ITG linear growth rate $\gamma_{\mathrm{ITG}}$. The shearing rate $\omega_{E \times B}$ is defined as $[15,16]$ 


$$
\omega_{E \times B}=\left(\frac{\Delta \psi_{0}}{\Delta \phi_{0}}\right)\left|\frac{\partial^{2} \Phi_{0}(\psi)}{\partial \psi^{2}}\right| \cong\left|\frac{R B_{\theta}}{B_{\phi}} \frac{\partial}{\partial r}\left(\frac{E_{r}}{R B_{\theta}}\right)\right|,
$$

where $\Delta \psi_{0}$ and $\Delta \phi_{0}$ are the correlation lengths of the ambient turbulence in the radial and toroidal directions, and $\Phi_{0}, E_{r}, B_{\theta}$, and $B_{\phi}$ are the equilibrium electrostatic potential, radial electric field, and poloidal and toroidal magnetic fields, respectively. In helical plasmas, the radial electric filed $E_{r}$ is determined from the ambipolarity condition of helical ripple-induced neoclassical flux, whereas in tokamaks, $E_{r}$ is not easily determined, but can be calculated from the plasma radial force balance equation under the assumption that the poloidal velocities can be expressed according to the neoclassical theory $[17,18]$. However, in this paper, $E_{r}$ is described simply as

$$
\frac{\mathrm{d} E_{r}}{\mathrm{~d} r} \cong-\frac{1}{e n_{\mathrm{i}}^{2}} \frac{\mathrm{d} n_{\mathrm{i}}}{\mathrm{d} r} \frac{\mathrm{d} p_{\mathrm{i}}}{\mathrm{d} r},
$$

in the H-mode condition [19], where $n_{\mathrm{i}}$ and $p_{\mathrm{i}}$ are ion density and ion pressure, respectively. The ITG growth rate $\gamma_{\mathrm{ITG}}$ is defined as [20]

$$
\gamma_{\mathrm{ITG}}=\frac{\left(\eta_{\mathrm{i}}-2 / 3\right)^{1 / 2}|s| c_{\mathrm{i}}}{q R}
$$

where $\eta_{\mathrm{i}}=L_{\mathrm{n}} / L_{\mathrm{T}}, c_{\mathrm{i}}=\sqrt{T_{\mathrm{i}} / m_{\mathrm{i}}}$, and $s$ is the magnetic shear defined as

$$
s=\frac{r}{q}\left(\frac{\mathrm{d} q}{\mathrm{~d} r}\right) .
$$

Most theoretical studies based on the $E \times B$ shear stabilization adopt a thermal diffusion coefficient $\chi$ in the form

$$
\begin{aligned}
\chi_{\mathrm{e}, \mathrm{i}}=\chi_{\text {neoclassical }}+\chi_{\text {anomalous }}, & \\
\chi_{\text {anomalous }}= & \alpha_{1} \times \chi_{\text {Gyrobohm }} \\
& +\alpha_{2} \times \chi_{\text {Bohm }} \times F\left(\omega_{E \times B} t / \gamma_{\mathrm{ITG}}\right),
\end{aligned}
$$

or

$$
\begin{aligned}
\chi_{\text {anomalous }}= & \left(\alpha_{1} \times \chi_{\text {Gyrobohm }}+\alpha_{2} \times \chi_{\text {Bohm }}\right), \\
& \times F\left(\omega_{E \times B} / \gamma_{\mathrm{ITG}}\right),
\end{aligned}
$$

where

$$
F\left(\omega_{E \times B} / \gamma_{\mathrm{ITG}}\right)=\frac{1}{1+\left(\omega_{E \times B} / \gamma_{\mathrm{ITG}}\right)^{2}} .
$$

The coefficient $\chi$ neoclassical is the neoclassical part of the thermal diffusion coefficient, and $\chi$ anomalous is the anomalous part described in the Bohm and GyroBohm mixed transport model $[11,12]$. In this paper, equation (6) is used in the tokamak case with fitting parameters $\alpha_{1}=0.5$ and $\alpha_{2}=4.0$ obtained from ref [12], and equation (7) is adopted in the helical case with $\alpha_{1}=5.0$ and $\alpha_{2}=0.2$ decided from the comparison between the LHD experimental data and the simulation using TOTAL code.

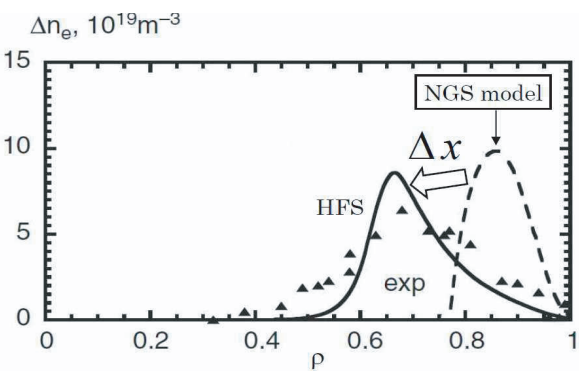

Fig. 1 Schematic of calculation of HFS pellet injection. The ablation profile is calculated using the standard NGS model; if is shifted toward the direction to the center by $\Delta R$ calculated using the mass relocation model $\left(\Delta n_{\mathrm{e}}\right.$ is density deposition, $\rho$ is normalized radius).

\subsection{HFS pellet injection}

HFS pellet injection is described as two processes: pellet ablation and mass relocation. We simulate HFS injection using the pellet penetration model combined with the ablation and mass relocation models. A schematic diagram is provided in Fig. 1. Pellet ablation models have been investigated by many authors, e.g., neutral gas shielding (NGS) model [1-4], neutral gas plus plasma shielding (NGPS) model [21-26], and two-dimensional lentil model [27] with current acceleration. Here, we use here the most widely adopted ablation model, NGS, which is a one-dimensional approach with a monoenergetic electron heat flux model based on steady-state approximations and on the assumption of spherically symmetric hydrodynamic expansion. The pellet ablation rate is described as

$$
\frac{\mathrm{d} N}{\mathrm{~d} t}=1.12 \cdot 10^{16} n_{\mathrm{e}}^{0.333} T_{\mathrm{e}}^{1.64} r_{\mathrm{p}}^{1.333} M_{\mathrm{i}}^{-0.333},
$$

where $N, n_{\mathrm{e}}, T_{\mathrm{e}}, r_{\mathrm{p}}$, and $M_{\mathrm{i}}$, are the number of particles in a pellet, the electron density, electron temperature, pellet radial size, and pellet mass respectively.

The mass relocation width from the ablation point with the plasmoid drift in the major-radius direction $\Delta x$ is described as [28]

$$
\begin{aligned}
\Delta x= & -0.5 q \beta B_{\mathrm{t}} B_{\mathrm{p}}^{-1}\left(1+q L_{\mathrm{e}} / a\right)^{-1} \\
& \times a^{-2} r_{0}^{2} \delta n(n+\langle\delta n\rangle)^{-1} .
\end{aligned}
$$

Based on the above, the deposition by the HFS pellet injection is described as

$$
\Delta n_{\mathrm{e}, \mathrm{HFS}}(\rho)=\Delta n_{\mathrm{e}, \mathrm{NGS}}(\rho+\Delta x)
$$

\section{Simulation Results}

\subsection{Demonstration of HFS pellet injection}

The reactor machine parameters used in this paper are shown in Table 1; they were typical designs optimized using the reactor design system code PEC (PhysicsEngineering-Cost) [14]. These parameters are derived from two 1-GW electric power fusion reactor designs: 
Table 1 Reactor machine parameters used in this paper

\begin{tabular}{lll}
\hline & $\begin{array}{l}\text { Helical reactor } \\
\text { (LHD-like) }\end{array}$ & $\begin{array}{l}\text { Tokamak reactor } \\
\text { (ITER-like) }\end{array}$ \\
\hline$R_{\mathrm{p}}[\mathrm{m}]$ & 12.5 & 5.2 \\
$a_{\mathrm{p}}[\mathrm{m}]$ & 2.2 & 1.7 \\
$\kappa$ & - & 2.0 \\
$\delta$ & - & 0.5 \\
$B_{\mathrm{t}}[\mathrm{T}]$ & 4.6 & 7.1 \\
\hline
\end{tabular}

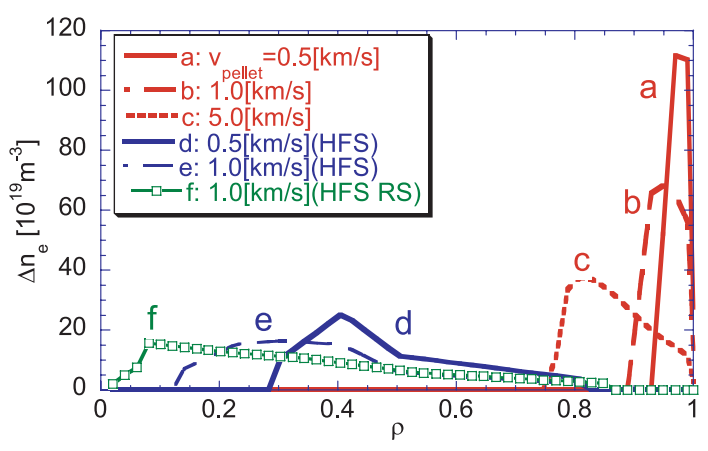

Fig. 2 Model prediction for the HFS pellet injection in a tokamak reactor. Ablation profiles are shown by a, b, and c, and HFS injection simulation results are given by d, e, and $f$ depending on the pellet injection velocities. The profiles $\mathrm{d}$ and $\mathrm{e}$ are in the normal shear case, and $\mathrm{f}$ is in the reversed-shear case (pellet size $=5 \mathrm{~mm}$ ).

high-field, high- $\beta$ compact tokamak reactor TR-1 and high-beta helical system HR-1.

Typical results of simulation of HFS pellet injection in a tokamak reactor are shown in Fig. 2. The pellet ablation densities are shown for different pellet injection velocities. Here, the radial parabolic temperature and flat density profiles are assumed as $T(x)=T_{0}\left(1-x^{2}\right)^{3}$ and $n(x)=n_{0}\left(1-x^{2}\right)^{0.5}$, with $T_{0}=30 \mathrm{keV}$ and $<n>=10^{20} \mathrm{~m}^{-3}$. This figure shows that HFS injection could provide significantly deep fuelling in reactor-grade tokamak plasmas, and further increase in the injection velocity improves central fuelling. For assumed temperature and density profiles, HFS injection with a pellet velocity of $1 \mathrm{~km} / \mathrm{s}$ could provide density increase at the normalized radius $\rho \sim 0.1$. Moreover, it shows that the reversed-shear mode improves central fuelling for HFS injection based on the mass relocation model of equation (10).

\subsection{ITB simulation in tokamak reactors}

In the previous subsection, we show that pellet injection could provide deep fuelling in tokamak reactors using HFS injection. Here, we consider an operation scenario with ITB formation in the deep penetration case (using HFS injection) and shallow penetration case (using medium-field side injection) in reversed-shear ITB plasmas.

In this simulation, pellet injection flux is assumed continuous, and for burning control, alpha particle power and
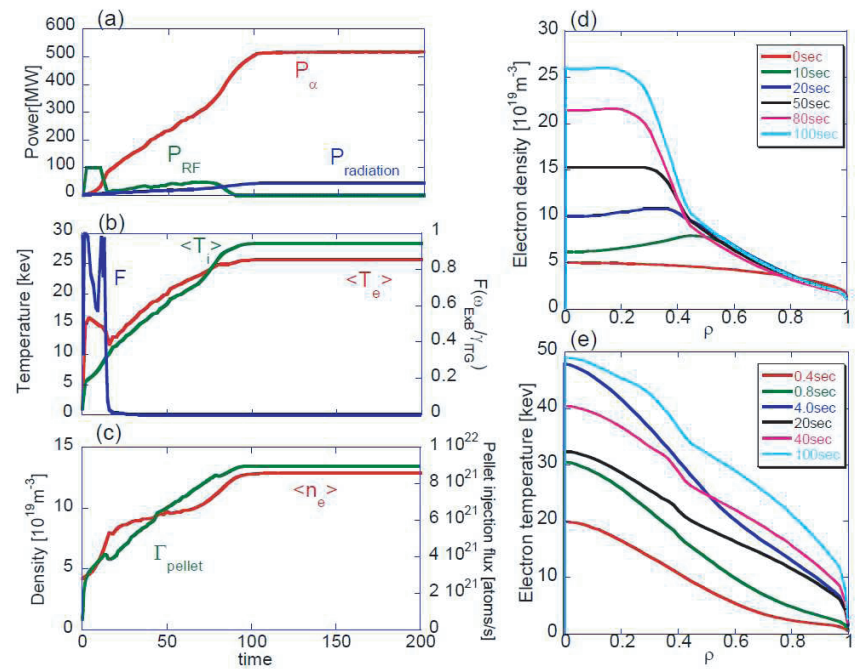

Fig. 3 (a), (b), and (c) show the operation scenarios of TR-1 (reversed-shear) with HFS pellet injection. The improvement factor $\mathrm{F}\left(\omega_{E \times B} / \gamma_{\mathrm{ITG}}\right)$, which is defined in equation (8), is the value at $\rho=0.4$. Figure (d) and (e) show the time evolution of the electron density and temperature radial profiles.

plasma density are feedback-controlled by adjusting both heating power and fuelling. The q-profile is the input parameter $\left(q_{\mathrm{axi}}=2.5, q_{\mathrm{min}}=2.4, q_{\mathrm{sur}}=3.2\right)$, and the total plasma current is fixed $\left(I_{\text {total }}=14.7 \mathrm{MA}\right)$. The bootstrap current is calculated by the NCLASS code [30] part in the TOTAL code. The required externally driven current density $j_{\text {external }}$ is described as

$$
j_{\text {external }}=j_{\text {total }}-j_{\mathrm{BS}} .
$$

Figure 3 shows the operation scenarios of the tokamak reactor (reversed-shear mode) in the deep penetration case using HFS pellet injection. In this scenario, ITB is formed at $15 \mathrm{sec}$ and plasma is ignited at $100 \mathrm{sec}$.

Figure 4 shows comparisons with radial profiles in the steady state (time $=200 \mathrm{sec}$ ) in deep (same as Fig. 3) and shallow cases. We can see that there are clear differences between both cases. In the deep penetration case shown on the left of Fig. 4, an ITB is formed at $\rho \sim 0.4$, but in the shallow case on the right, it is not.

The reason is shown in the bottom two figures in Fig. 4. In this simulation, ITB formation is determined by two parameters, $\omega_{E \times B}$ and $\gamma_{\mathrm{ITG}}$. In both cases, $\gamma_{\mathrm{ITG}}$ is reduced at $\rho \sim 0.4$ where the magnetic shear $s \sim 0$. Suppression occurs when $\omega_{E \times B}$ exceeds $\gamma_{\mathrm{ITG}}$; therefore an ITB tends to be formed at a low $\gamma_{\mathrm{ITG}}$ position. However, in the shallow penetration case, the shearing rate $\omega_{E \times B}$ is small at $\rho \sim 0.4$, because the gradient of the radial electric field $\mathrm{d} E_{r} / \mathrm{d} r$ is small depending on the term $\mathrm{d} n_{\mathrm{i}} / \mathrm{d} r$ in equation (2). The transient density profile and relevant clear ITB formation depend on the pellet penetration depth. Deeper pellet penetration results in a steeper gradient of $E_{r}$ and a larger shearing rate $\omega_{E \times B}$ at the position of small $\gamma_{\mathrm{ITG}}$, so 

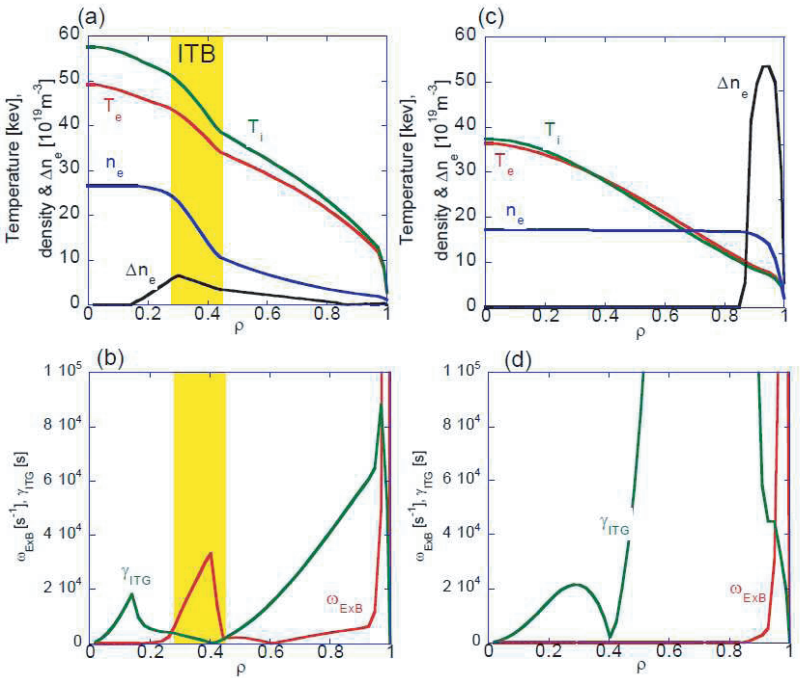

Fig. 4 ITB simulation results with pellet injection in the reversed-shear tokamak reactor. Figures on the left denote the deep pellet penetration case (using HFS), and figures on the right are the shallow penetration case (using the medium-field side injection). The upper figures (a) and (c) show ion and electron temperatures, electron density, and pellet deposition profiles, and the lower figures (b) and (d) show $\omega_{E \times B}$ and $\gamma_{\mathrm{ITG}}$ profiles.

that an ITB is formed there. We can say that deep pellet penetration plays an important role in ITB formation, and HFS pellet injection is considerably effective as a technique for confinement improvement as well as core density fuelling in the reversed-shear tokamak reactor.

\subsection{ITB simulation in helical reactors}

Figure 5 shows the results of ITB simulation in a helical reactor with the operation scenario similar to the tokamak reactor (ignition at time $=80 \mathrm{sec}$ and $P_{\alpha}=450 \mathrm{MW}$ in the steady state). In this simulation, which is different from the tokamak case, the method for achieving the central fuelling (pellet penetration depth $\lambda_{\text {pellet }}<0.1$ ) corresponds to high-speed pellet injection and not to HFS injection, because the effectiveness of HFS injection has not been observed so far in a helical system [29]. This might be because the field connection length between the high-field side and the low field side is quite short in comparison with the tokamak case.

In Fig. 5 (b), we can see that the improvement factor $F$ defined in equation (8) is reduced at $\rho<0.2$, and it causes improvement in anomalous transport, resulting in the formation of ITB. Moreover, from Fig. 6, it can be seen that the peaked density profile with deep pellet penetration tends to cause strong reduction in the improvement factor $F$. Therefore, these results show that deeper pellet penetration is more effective for ITB formation in helical reactors. However, in order to attain central fuelling $\left(\lambda_{\text {pellet }}<0.1\right)$, a pellet injection velocity of about $50 \mathrm{~km} / \mathrm{s}$ is required (at
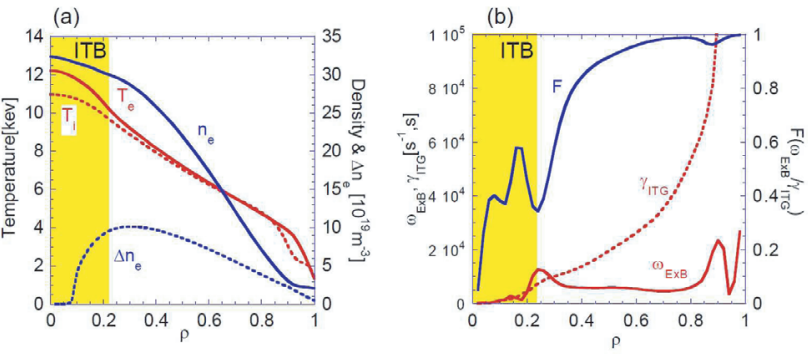

Fig. 5 ITB simulation results with pellet injection in helical reactor. Temperature, density, and deposition profiles are shown in figure (a), and $\omega_{E \times B}, \gamma_{\mathrm{ITG}}$, and improvement factor $\mathrm{F}\left(\omega_{E \times B} / \gamma_{\mathrm{ITG}}\right)$ defined in the equation (8) are shown in figure (b).
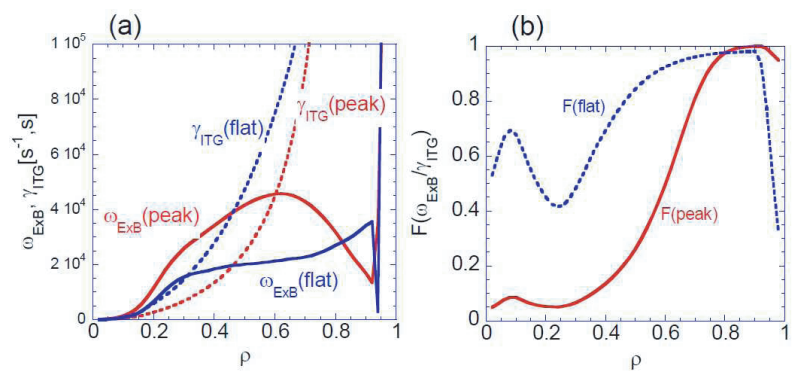

Fig. 6 Comparison of peak and flat density profile cases. $\omega_{E \times B}$, and $\gamma_{\text {ITG }}$ are shown in (a), and improvement factor $F$ is shown in (b).

pellet size $=4 \mathrm{~mm}$ ), which is an unrealistic value. In order to realize the scenario in which an ITB is formed with peaked density profile by central fuelling, methods other than pellet injection may be needed in a helical reactor.

\section{Conclusion}

We investigated the relationship between ITB formation and pellet injection in tokamak and helical reactors. HFS pellet injection in a tokamak reactor was analyzed and its effectiveness was clarified. In a tokamak reactor with a reversed-shear profile, it was shown that the pellet penetration depth plays an important role in ITB formation, and HFS injection would be an effective technique for confinement improvement as well as plasma core fuelling. In addition, we showed that deeper pellet penetration is more effective for ITB formation in a helical reactor. However, very-high-speed pellet injection is required in order to realize central fuelling. Therefore, for ITB formation in a helical reactor, other fuelling techniques, such as HFS pellet injection in tokamak, may be needed.

[1] P. Parks et al., Nucl. Fusion 17, 539 (1977).

[2] S. Milora and C. Poster, IEEE Trans. Plasma Sci. PS-6, 578 (1978).

[3] P.B. Parks and R.J. Turnbull, Phys. Fluids 21, 1735 (1978). 
[4] L.R. Bayler et al., Nucl. Fusion 32, 2177 (1992).

[5] P.T. Lang et al., Phys. Rev. Lett. 79, 1478 (1997).

[6] L.R. Bayler et al., Fusion Technol. 34, 425 (1998).

[7] S. Ishida et al., Proc. 14th Int. Conf. Plasma Physics Controlled Nuclear Fusion Research (Würzburg, 1992) vol 1 (Vienna: IAEA) 219 (1993).

[8] H. Kimura et al., Phys. Plasmas 3, 1943 (1996).

[9] JET Team (presented by Schmidt G L) Proc. 12th Int. Conf. Plasma Physics and Controlled Nuclear Fusion Research (Nice, 1988) vol 1 (Vienna: IAEA) 215 (1989).

[10] B. Tubbing et al., Nucl. Fusion 31, 839 (1991).

[11] J. Garcia, K. Yamazaki, J. Dies and J. Izquierdo, Phys. Rev. Lett. 96, 105007 (2006).

[12] T. Tala et al., Plasma Phys. Control. Fusion 43, 507 (2001).

[13] K. Yamazaki and T. Amano, Nucl. Fusion 32, 633 (1992).

[14] K. Yamazaki et al., Fus. Eng. Des. 81, 2743 (2006).

[15] T.S. Hahm and K.H. Burrell, Phys. Plasmas 2, 1648 (1995).

[16] P. Zhou, W. Horton and H. Sugama, Phys. Plasmas 6, 2503 (1999).
[17] D.R. Ernst et al., Phys. Plasmas 7, 615 (2000).

[18] Y.B. Kim, P.H. Diamond and R.J. Groebner, Phys. Fluids B 3, 2050 (1991).

[19] F.L. Hinton and G.M. Staebler, Phys. Fluids B 5, 1281 (1993)

[20] B. Esposito et al., Plasma Phys. Control. Fusion 45, 933 (2003).

[21] M. Gouge et al., Fusion Technol. 19, 95 (1991).

[22] W. Houlberg et al., Nucl. Fusion 28, 595 (1988).

[23] W. Houlberg et al., Nucl. Fusion 32, 1951 (1992).

[24] M. Kaufmann et al., Nucl. Fusion 26, 171 (1986).

[25] P. Parks Nucl. Fusion 32, 2137 (1992).

[26] B. Pegourie et al., Nucl. Fusion 33, 591 (1993).

[27] B. Kuteev Nucl. Fusion 35, 431 (1995).

[28] A.R. Polevoi and M. Shimada, Plasma Phys. Control. Fusion 43, 1525 (2001).

[29] R. Sakamoto et al., Proc. 29th EPS Conf. Plasma Phys. Contr. Fusion, (Montreux, 2002), ECA Vol.26B, P-1.074 (2002).

[30] W.A. Houlberg et al., Phys. Plasma 4, 3230 (1997). 